POPULATION STUDY ARTICLE

\title{
Prospective associations between television in the preschool bedroom and later bio-psycho-social risks
}

\author{
Linda S. Pagani ${ }^{1}$, Marie Josée Harbec ${ }^{2}$ and Tracie A. Barnett ${ }^{3}$
}

\begin{abstract}
BACKGROUND: North American child media guidelines suggest screen-free zones without offering clear evidence and alternative harm-reduction strategies. Our hypothesis is that having a bedroom television during the preschool years will be prospectively associated with mental and physical health risks in adolescence.

METHODS: Participants are from a prospective-longitudinal birth cohort of 907 girls and 952 boys from the Quebec Longitudinal Study of Child Development. Child outcomes at ages 12 and 13, measured by multiple sources, were linearly regressed on having a bedroom television at age 4 .

RESULTS: Bedroom television at age 4 predicted a higher body mass index at age 12 (standardized $B=0.10, p<0.001$ ), more unhealthy eating habits at age $13(B=0.10, p<0.001)$, higher levels of emotional distress $(B=0.12, p<0.001)$, depressive symptoms $(B=0.08, p<0.001)$, victimization $(B=0.07, p<0.001)$, physical aggression $(B=0.09, p<0.001)$, and lowers levels of sociability $(B=-0.09, p<0.001)$ at age 12 , above and beyond pre-existing individual and family factors.

CONCLUSION: The bedroom as a screen-based preschool zone does not bode well for long-term cardio-metabolic wellness, mental health, and social relationships.
\end{abstract}

Pediatric Research (2019) 85:967-973; https://doi.org/10.1038/s41390-018-0265-8

\section{INTRODUCTION}

How children spend their leisure time influences their long-term well-being. ${ }^{1}$ Despite other more current forms of media, television and movies remain the most common pastime for young children. ${ }^{2,3}$ There is developmental persistence in how youth invest their discretionary time, as child televiewing at age 2 predicts significant preschool increases in televiewing ${ }^{4}$ and overall media use in adolescence. ${ }^{5,6}$ Cumulative hours of screen use in childhood and adolescence imply its constant input during growth and development, especially if it is more accessible, as in a bedroom. ${ }^{2,7}$

Because it is a lifestyle habit from preschool onward, there are bio-psycho-social concerns regarding watching too much television in early childhood. ${ }^{2,7}$ Using a birth cohort born at a time when the only media available was on television screens (broadcast, videocassettes, and DVDs), we found prospective associations between early childhood televiewing and body mass index, physical sedentariness and fitness, and eating habits characterized by more soft drink consumption and sweet snacking and less fruit and vegetable intake by preadolescence. ${ }^{4,8}$ Such characteristics induce biological consequences over the life course, especially cardiometabolic risk. Early childhood televiewing was also prospectively associated with later cognitive skills ${ }^{4,9}$ and social impairment. ${ }^{5}$ Of particular concern is the finding between early childhood televiewing and subsequent victimization. ${ }^{4-6,9}$

Too much discretionary screen exposure likely displaces time for other more enriching physical and mental activity, with concomitant long-term risks for physical activity, brain plasticity, and socio-emotional intelligence. ${ }^{10,11}$ Cortical development during the preschool period is characterized by exuberant synaptogenesis and subsequent pruning to improve brain connectivity, which is mainly driven by social interaction. ${ }^{12}$ By honing a synchronized orchestra of coordinated functions in brain plasticity, human physical, and social interaction provide stimulation for cognitive, motor, and emotional growth and development. ${ }^{13}$ It is not surprising that cumulative hours of discretionary screen time are associated with less effective executive processes that govern decision-making. ${ }^{12}$ Ineffective mental governance promotes less healthy behavioral dispositions and routines. ${ }^{10}$ Thus, excessive discretionary screen time likely creates a time debt for actively polishing developmental skills and may explain, in part, the risks of poor physical and mental health and social and academic impairment in middle childhood. ${ }^{4}$

In response to the body of evidence showing dose-response relationships, North American daily guidelines for parents underscore limits of not more than 1 hour of screen media exposure from ages 2-5 and not more than 2 hours for school age children, assuming that the content is developmentally appropriate. ${ }^{2,3,7}$ These guidelines also emphasize the importance of media-free locations and activities in the family home. Parents are encouraged to keep bedrooms free of screens. ${ }^{2,3,7}$ Easy access often translates to more exposure, which in turn begets the concomitant of excessive discretionary screen time. ${ }^{10,14}$ The reasoning is that easy media access may impede adequate sleep, physical activity, and other healthy lifestyle behaviors such as eating in interactive dining areas with other family members. ${ }^{2,3,7,14,15}$ Bedroom placement of screens may therefore harbor cardiometabolic risks. ${ }^{14,16,17}$ Nevertheless, 17 and $23 \%$ of children, respectively aged 4 and 7 , have a bedroom television. ${ }^{16,17}$ From ages 10-14, estimates rise to almost two-thirds, and are expected

\footnotetext{
${ }^{1}$ School of Psycho-Education, Université de Montréal, Montréal, Canada; ${ }^{2}$ School Environment Research Group, Université de Montréal, Montréal, Canada and ${ }^{3}$ Sainte-Justine's Hospital Research Center, Montréal, Canada

Correspondence: Linda S. Pagani (Linda.s.pagani@umontreal.ca)
}

Received: 20 August 2018 Revised: 7 November 2018 Accepted: 9 November 2018

Published online: 26 December 2018 
to persist. $^{18}$ Given the estimated consequences of excessive discretionary screen time on later bio-psycho-social outcomes, television placement in youthful bedrooms may not be a healthy choice for reasons other than metabolic risk.

Theoretically, a bedroom represents a highly personalized domestic space for essential and non-essential developmentally appropriate human endeavors such as sleep and leisure activity. ${ }^{19}$ It represents a designed space in which adults/ children live, play/work, and recreate daily. ${ }^{20,21}$ Regardless of indoor or outdoor status, human spaces can either promote or impede physical and social human activity, affecting both physical and mental health across the population. ${ }^{20-22}$ Time spent indoors is characteristically more personal and less active than outdoors. ${ }^{22}$ Children spend much of their waking time sitting indoors, and time spent indoors is often inactive, related to screens, and spent alone. ${ }^{23}$ Consequently, children might be vulnerable to the conditions and limitations of their interior, more personalized space ${ }^{24}$ and this vulnerability may translate into long-term outcomes. Beyond the obesogenic risk, the North American bedroom guidelines warrant more empirical support. Using longitudinal data, Gentile et al. ${ }^{25}$ concluded that bedroom media represent a robust risk factor for several aspects of child development such as reading, less sleep, and physical activity, and more exposure to media violence. These might explain subsequent risks for academic performance, obesity, and physical aggression outcomes.

A comprehensive study that prospectively examines long-term bio-psycho-social risks; that is, above and beyond pre-existing individual and family factors, would result in specific compelling reasons for avoiding bedroom placement of screens.

In the present study, we use the Quebec Longitudinal Study of Child Development (QLSCD) birth cohort to examine prospective associations between having a bedroom television during the neurodevelopmentally critical preschool period and subsequent bio-psycho-social lifestyle risks in early adolescence. Measures comprise information from parent, teacher, and direct child assessments. It is hypothesized that having a bedroom television will be prospectively associated with unhealthy physical, mental, and social outcomes beyond the competing influence of individual and family confounders, including overall screen media use in early adolescence.

\section{METHODS}

\section{Participants}

Coordinated by the Institut de la Statistique du Québec, the QLSCD originates from a randomly selected, stratified sample of 2837 infants born between 1997 and 1998 in Quebec, Canada. ${ }^{26}$ The children were selected using the birth register of Quebec. The main objective of this study is to provide data on the development of typically developing children. At the inception of the longitudinal component, 93 children were deemed ineligible and 172 were untraceable owing to incorrect coordinates. Of the 2572 remaining children, some parents were unreachable or refused participation. Thus, 2223 5-month-old infants (and their families) with parental consent were deemed eligible for annual follow-up from 5 months onward, representing $82 \%$ of the eligible target population. Of these, 39\% were firstborn. Biennial follow-up occurred during the school age years. Participants were included in this IRB-approved study if they had completed child reports on having a bedroom television at age 4 ( $n=1859$ out of 2223). Outcome variables, based on quality and availability, were measured at ages 12 and 13 .

\section{Measures \\ Preschool bedroom predictor (age 4.17 years, $S D=0.26$ ). Inter- viewer asked the child if he had a television in his bedroom $(0=$} no and $1=$ yes). Parents reported daily television exposure during weekdays and weekends at age 4 . This measure included six response choices: $1=0 \mathrm{~h}(1.1 \%), 2=$ less than $1 \mathrm{~h}(30.5 \%), 3=1$ to $3 \mathrm{~h}(59.4 \%), 4=3$ to $5 \mathrm{~h}(7.3 \%), 5=5$ to $7 \mathrm{~h}(1.3 \%)$, and $6=$ more than $7 \mathrm{~h}(0.5 \%)$. Noteworthy is that $68.5 \%$ of the children viewed more than one hour of television daily, which means they would have exceeded the recommendations of not more than one hour per day between age 2 and 5 . $^{2}$ There was a positive correlation between having a bedroom television and daily television exposure, $r=0.11, p=0.000$. Children without a bedroom television watched an average of $1 \mathrm{~h} 44 \mathrm{~min}$ of daily television, and those with a bedroom television watched an average of $2 \mathrm{~h}$ $10 \mathrm{~min}$ of daily television.

Bio-psycho-social risks outcomes (13.13 years, $S D=0.26)$

Biological risks: Measures of body mass index (BMl; calculated as weight in kilograms divided by height in meters squared) were taken by trained independent examiners who followed a standardized protocol detailed elsewhere. ${ }^{27}$ One year later, we obtained adolescent self-reports of their dietary consumption of unhealthy foods, with responses ranging from 0 (never) to 7 (4 times or more per day). We derived a scale comprising 10 items: French fries; white bread; delicatessen; soft drinks; diet drinks; fruit flavored drinks; sports drinks; energy drinks; sweet snacks; and candies; $a=0.82$. Higher responses on this measure indicate a higher energy dense and nutrient poor intake. ${ }^{2,29}$

Psychological risks: Sixth grade teachers rated emotional distress ( 5 items: seems to be unhappy or sad; is not as happy as other children; has no energy, is feeling tired; has trouble enjoying himself or herself; and is unable to make decisions; $a=$ 79). This factor was rated on a Likert scale with response options including 1 (never or not true), 2 (sometimes or somewhat true), and 3 (often or very true). Factor scores were then rescaled from 0 to 10 , with higher values indicating more emotional distress. Youths also completed a short version of the Children's Depression Inventory (CDI, 10 items): personal achievement; self-esteem; feelings of loneliness; feelings of being loved; feelings of sadness; hope for the future; feel like crying; bothered by something; perception of physical appearance; and number of friends; $a=79 .{ }^{30}$ Higher values indicate a higher degree of depressive symptomatology.

Social risks: Sixth-grade teachers reported on child sociability (3 items: works cooperatively; plays cooperatively; and demonstrates self-confidence; $a=77$ ), victimization ( 3 items: insulted by peers; laughed at; and pushed, shoved, or hassled; $a=80$ ), and physical aggression ( 3 items: physically attacks others; fights; and hits/ bites/gives kicks; $a=85$ ). These factors were rated on a Likert scale with response options including 1 (never or not true), 2 (sometimes or somewhat true), and 3 (often or very true). Factor scores were then rescaled from 0 to 10 , with higher values indicating a higher degree of social impairment. These factors represent robust predictors of future social adjustment and school success. ${ }^{4,5,9}$

Pre-existing and concurrent control variables (5 months to 12 years, risk Category $=1$, no risk $=0$ ). Individual characteristics include child sex (boys $=1$ ), temperament (age 1.5, both parent-reports summed, difficult and unpredictable temperament $=1$ ), early cognitive skills (age 2, Imitation Sorting Task which assesses attention and working memory, below median $=1$ ), directly measured child BMI (age 2, for analyses involving BMI and unhealthy eating habits outcomes), parent-reports on emotional distress and physical aggression for analyses involving psychological and social risks outcomes (age 2), and self-reported concurrent discretionary screen time (hours of television, computer, and videogame exposure during both week and weekend at age 12). Maternal education at 5 months, parent-reported family 
dysfunction at (with lower scores revealing that a family is functional) and directly measured maternal BMl at age 1.5 for analyses involving BMI and unhealthy eating habits outcomes at age $1.5^{8}$ family configuration (age 2 , two-parent $=0$ and singleparent $=1$ ), and parental antisocial behavior during adolescence and adulthood measured (5 months), for analyses involving psychological and social risks outcomes, using a composite score from mother and father responses to the National Institute of Mental Health-Diagnostic Interview Schedule. Higher scores correspond to more parental antisocial behavior and correlate with social and occupational impairment. ${ }^{31}$

Data analytic strategy

In this study, we aimed to examine long-term prospective linear associations using OLS multiple regression, in which indicators of bio-psycho-social risk outcomes at ages 12 and 13 years are regressed on having a bedroom television at age 4 . To reduce the possibility of omitted variable bias, we estimate this linear relationship while simultaneously controlling for child characteristics (sex; temperament problems at age 1.5; cognitive skills at age 2; BMl at age 2; SBQ scores at age 2 on emotional distress and physical aggression; and total hours of discretionary screen time per week at age 12) and family characteristics (maternal education at 5 months; family dysfunction at age 1.5; maternal BMI at age 1.5; family configuration at age 2; and parental antisocial behavior during adolescence and adulthood).

This study required follow-up data from several sources and waves. As with any longitudinal study, incomplete data required an attrition analysis to compare the participants with varying incomplete data on control variables to participants with complete data on control variables from our sample (see online appendix). Incomplete data were not missing at random. We conducted multiple imputation in SPSS to correct for response and attrition bias. $^{32}$ Using a stochastic algorithm, missing observations are imputed based on available complete data on auxiliary variables, creating multiple datasets that are copies of the original complete data. The algorithm generates slightly different values for each imputed measure across the multiple datasets. The additional variance caused by differences in imputed values between the various copies reflects the uncertainty of the imputation and is added as a correction to the analyses. ${ }^{32}$

\section{RESULTS}

Table 1 provides descriptive statistics of predictor, outcomes, and control variables. Boys comprised half the sample. Only $12 \%$ lived in single-parent homes at age 2 . By age $4,13 \%$ of the participants had a television in their bedroom. At age 12, 37\% of the children had a bedroom television. There was a positive correlation between having a bedroom television at age 4 and having one at age $12, r=0.23, p=0.00$. At age $6,68 \%$ of the families ate together every day (not shown). Television exposure by age 2 averaged $8.82 \mathrm{~h}$ for the entire week $(S D=6.17)$ and rose to an average of 14.85 per week by age $4(S D=8.05)$ and then diminished to an average of $12.13 \mathrm{~h}$ per week of total discretionary screen time, give or take $\sim 10 \mathrm{~h}$ across participants by age 12 (not shown). More specifically, by age 12, 25\% of boys and $38 \%$ of girls reported using more than $14 \mathrm{~h}$ of discretionary screen time (television, computer, and videogame exposure) weekly, thus exceeding quantities in recommendations of not more than 2 hours per day beyond age $5 .^{7}$ Weekend television was more frequent and intense. White bread $(M=2.29)$ was the most consumed unhealthy food, whereas energy drinks were the least consumed $(M=0.24)$. According to their BMI, $12 \%$ of the children at age 12 were classified as overweight (85th to 97 th percentile) and $3 \%$ were classified as obese or severely obese (above the 97th percentile).
Table 2 documents the relationship between the pre-existing controls and having a bedroom television at age 4 . Boys had more probabilities of having a bedroom television at age 4 (standardized $B=0.06, p<0.001$ ). Higher levels of temperament problems at age 1.5 (standardized $B=-0.05, p<0.001$ ), and higher levels of cognitive ability (standardized $B=-0.01, p<$ 0.001 ) and emotional distress at age 2 (standardized $B=-0.01$, $p<0.01$ ) predicted lower probabilities of having a bedroom television at age 4. A higher BMI (standardized $B=0.05, p<0.001$ ) and higher levels of physical aggression at age 2 (standardized $B=0.01, p<0.001$ ) predicted higher probabilities of having a bedroom television at age 4 . Also, mothers who did not finish high school when the child was aged 5 months (standardized $B=0.12$, $p<0.001$ ) and single-parent families (standardized $B=0.01, p<$ 0.001 ) predicted higher probabilities of having a bedroom television at age 4. Finally, higher levels of maternal BMI (standardized $B=0.03, p<0.001$ ), parental antisocial behavior (standardized $B=0.01, p<0.001$ ), and family dysfunction at age 1.5 (standardized $B=0.06, p<0.001$ ) predicted higher probabilities of having a bedroom television at age 4 .

Table 3 reports the relationship between having a bedroom television at age 4 and later independently measured and child reported biological risks. Having a bedroom television at age 4 predicted a higher $\mathrm{BMI}$ at age 12 (standardized $B=0.10, p<0.001$, Cohen's $f^{2}=0.18$ ), and more self-reported unhealthy eating habits at age 13 (standardized $B=0.10, p<0.001$, Cohen's $f^{2}=0.10$ ).

Table 4 reports the relationship between having a bedroom television at age 4 and teacher and child reported psychological and social risks at age 12 . Having a bedroom television at age 4 predicted higher levels of self-reported depressive symptoms (standardized $B=0.08, p<0.001$, Cohen's $f^{2}=0.05$ ), and teacherreported emotional distress (standardized $B=0.12, p<0.001$, Cohen's $f^{2}=0.08$ ), victimization (standardized $B=0.07, p<0.001$, Cohen's $f^{2}=0.07$ ), physical aggression (standardized $B=0.09, p<$ 0.001 , Cohen's $f^{2}=0.14$ ), and lower levels of sociability (standardized $B=-0.09, p<0.001$, Cohen's $f^{2}=0.14$ ) at age 12 . We found no interactions between the control and outcome variables.

\section{DISCUSSION}

Providing a bedroom television for children seemingly encourages personal and exclusive use. With media access, a child's bedroom becomes a central space where the child will be more likely to centralize daily living, eating, and recreating. ${ }^{14}$ Once time for nourishment, sleep, and hygiene are subtracted, the 24-hour day leaves limited time for honing cognitive, social, emotional, and physical skills. Easy accessibility to screens might create a time debt for other more enriching activity. Preschool bedroom television placement was associated with proportional increases in overall media use and bio-psycho-social risks to well-being by adolescence.

The findings suggest important prospective associations between preschool bedroom television and subsequent increased risks of unhealthy weight and consumption of energy dense, unhealthy foods such as fries, white bread, delicatessen, sugary drinks, sweet snacks, and candies. There are plausible explanations for these findings that go beyond less parental supervision. Compared with non-screen behaviors, screen-based activities increase dietary intake, even in the absence of television food advertising. ${ }^{33}$ Eating a meal alone during discretionary screen time is likely to reduce mindfulness about food consumption. ${ }^{34}$ Screen based consumption also predicts increased appetite after the meal itself. ${ }^{35}$ Our findings do not augur well given that, regardless of television placement, adolescents are already more sedentary and less structured by parents than younger children. ${ }^{36-38}$

Complementing these biological lifestyle risks, preschool bedroom television placement was more likely to be linked with 
Table 1. Descriptive statistics for the predictor, outcomes, and control variables

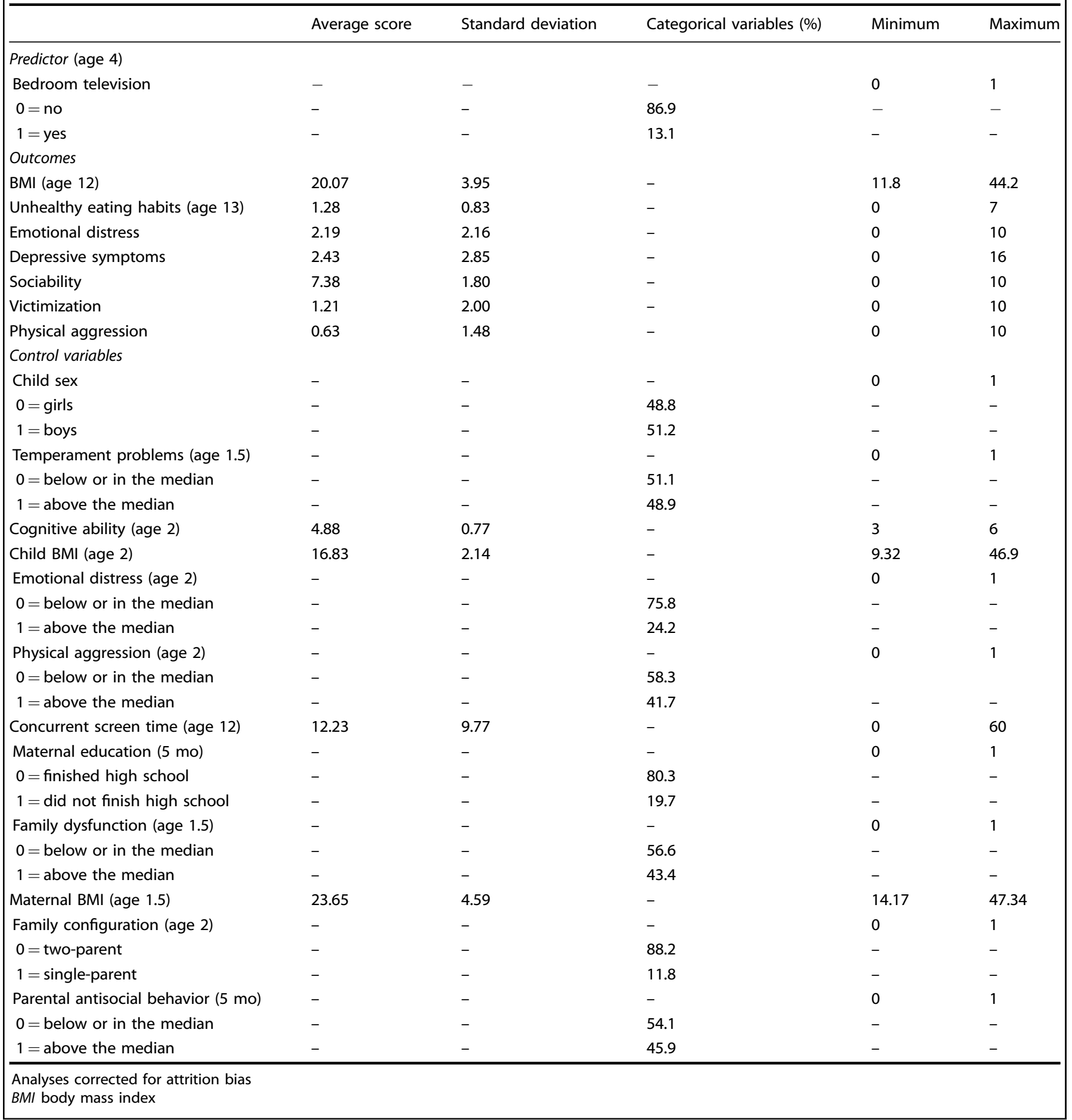

subsequent teacher observations of adolescent emotional distress. At the same assessment wave, youth with a preschool bedroom television were more likely to self-report loneliness, sadness, and negative feelings about their personal achievements, friends, self-esteem, physical appearance, and hope for the future, than their counterparts without a preschool bedroom television. Depressive symptoms that begin in childhood risk magnification by adolescence. ${ }^{39,40}$ Having a bedroom environment that encourages isolated and frequent televiewing seems to translate into a propensity for working less autonomously and cooperatively and confidently with classmates, compared to its absence.

We also observed a significant link with teacher-reported victimization. Preschool television forecasted middle school teacher observations of being laughed at or insulted and being pushed, shoved, or hassled by peers. Both findings directly relate to social and emotional intelligence. ${ }^{5}$ Taken together, these biopsycho-social outcomes could potentially transact and chart a life course path toward less mental and physical wellness over the long term. 
Table 2. Unstandardized regression coefficients (standard error) reflecting the adjusted relationship between baseline child and family characteristics between 5 months and age 2 and having a bedroom television at age 4

Baseline child and family control variables

$B$ (SE)

Bedroom television

Child sex

Temperament problems (age 1.5)

Cognitive ability (age 2)

Child BMI (age 2)

Emotional distress (age 2)

Physical aggression (age 2)

Maternal education (5 mo)

Family dysfunction (age 1.5)

Maternal BMI (age 1.5)

Family configuration (age 2)

Parental antisocial behavior (5 mo)

$0.04(0.001)^{* * * *}$

$-0.03(0.001)^{* * *}$

$-0.01(0.001)^{* * *}$

$0.01(0.000)^{* * * *}$

$-0.004(0.002)^{* *}$

$0.01(0.001)^{* * * *}$

$0.11(0.002)^{* * * *}$

$0.04(0.001)^{* * * *}$

$0.002(0.000)^{* * *}$

$0.01(0.002)^{* * *}$

$0.01(0.001)^{* * * *}$

Adjusted $R^{2}$

0.03

Analyses corrected for attrition bias

$B M I$ body mass index

${ }^{* *} p \leq 0.01,{ }^{* * *} p \leq 0.001$

Table 3. Unstandardized regression coefficients (standard error) reflecting the adjusted relationship between having a bedroom television at age 4 and biological risks at ages 12 and 13

\begin{tabular}{|c|c|c|}
\hline \multirow[b]{2}{*}{$\begin{array}{l}\text { Predictor and control } \\
\text { variables }\end{array}$} & \multicolumn{2}{|l|}{$B(\mathrm{SE})$} \\
\hline & BMI & $\begin{array}{l}\text { Unhealthy eating } \\
\text { habits }\end{array}$ \\
\hline Bedroom television (age 4) & $1.14(0.32)^{* * *}$ & $0.24(0.08)^{* * *}$ \\
\hline Child sex & $-0.57(0.21)^{* *}$ & $0.16(0.05)^{* *}$ \\
\hline $\begin{array}{l}\text { Temperament problems } \\
\text { (age 1.5) }\end{array}$ & $-0.54(0.20)^{* *}$ & $0.03(0.05)$ \\
\hline Cognitive ability (age 2) & $0.09(0.13)$ & $0.01(0.03)$ \\
\hline Child BMI (age 2) & $0.29(0.05)^{* * *}$ & $-0.02(0.01)$ \\
\hline $\begin{array}{l}\text { Concurrent screen time (age } \\
12 \text { ) }\end{array}$ & $0.03(0.01)^{*}$ & $0.01(0.003)^{* * *}$ \\
\hline Maternal education (5 mo) & $0.29(0.25)$ & $0.33(0.06)^{* * *}$ \\
\hline Family dysfunction (age 1.5) & $0.10(0.20)$ & $0.02(0.05)$ \\
\hline Maternal BMI (age 1.5) & $0.25(0.02)^{* * *}$ & $0.001(0.01)$ \\
\hline Family configuration (age 2 ) & $0.61(0.34)$ & $0.10(0.08)$ \\
\hline Adjusted $R^{2}$ & 0.16 & 0.08 \\
\hline \multicolumn{3}{|c|}{$\begin{array}{l}\text { Analyses corrected for attrition bias } \\
B M I \text { body mass index } \\
{ }^{*} p \leq 0.05,{ }^{* *} p \leq 0.01,{ }^{* * *} p \leq 0.001\end{array}$} \\
\hline
\end{tabular}

Learning during the preschool period is mainly driven by social interaction. Media access in the bedroom might also cut off face to face social interaction with family members during an important period of brain development related to emotional intelligence. ${ }^{10}$ The findings from this study indicate that availability and accessibility of television during early childhood generates a risk of life style habits and predispositions toward physical and mental illness. Specifically, bedroom access is associated with less engagement in effortful discipline and enrichment such as reading and sleep and physical activity, combined with increased risks of exposure to media violence. ${ }^{25}$ These factors explain subsequent propensities toward academic problems, unhealthy weight and dietary habits, and physical aggression.

Residential space likely provides a blueprint for the family environment and relationship proximity. Graham et al. ${ }^{19}$ offer six broad ambiance characteristics which explain how interior space might complement and humanize the family environment. A bedroom serves the following functions: ${ }^{1}$ restoration; ${ }^{2}$ kinship; ${ }^{3}$ storage; ${ }^{4}$ stimulation; ${ }^{5}$ intimacy; and ${ }^{6}$ productivity. Such ambiance characteristics during the preschool period would respectively involve ${ }^{1}$ sleep; ${ }^{2}$ togetherness; ${ }^{3}$ reading material and clothes; ${ }^{4}$ toys and intellectual tools (e.g., Lego, puzzles); ${ }^{5}$ parent-child and sibling-child discussions regarding thoughts and feelings; and ${ }^{6}$ learning tasks such as problem-solving and room-keeping. Too much access to one solitary space may thus limit interaction opportunities that are meant to enhance socio-emotional and behavioral intelligence and its regulation. Moreover, placement with private access does impair sleep hygiene, which can disrupt executive functions and socio-emotional regulation, increasing child behavior risks. ${ }^{25}$

This study reinforces the North American directives of mediafree periods and zones with the aim of increasing interaction and physical activity and limiting access to screen media in a preferred space. The bedroom as a screen-based preschool zone does not bode well for long-term cardio-metabolic wellness, mental health, and social relationships. These time-lagged associations were adjusted for pre-existing and concurrent alternative explanations that could have accounted for the observed links. The most important control was overall discretionary screen time at adolescence, because it was concurrent with the outcome variables. Although interesting and compelling, our observational study does not inform on individual processes related to invited and avoided interactions, solitude, and lack of parental supervision regarding personal dispositions (less optimal nutritional and sleep habits, maladaptive psychological responses, and social isolation). We are also unaware of meal time habits at the time of the bedroom television measure. These characteristics would require future in-depth analysis with primary data. We also know that parent-reported toddler televiewing averaged an hour and a half and rose to slightly over two hours per day during the preschool years ${ }^{4}$ and then diminished to under two hours, on average, self-reported by age $12 .^{6}$ It is most likely that the decrement by age 12 might be due to school schedule and homework and use of additional media devices and screen activities. On one hand, our retained "low risk" sample contained more children from two parent households and with mothers with higher educational attainment, which may limit the generalizability of our findings. On the other hand, the estimated risks can only be exacerbated in a higher risk sample. Although we can never be sure of residual or confounding bias, the analyses secured the predictor from an important number pre-existing and concurrent confounding influences, including self-reported discretionary screen time that was concurrently collected with the outcome data. Remarkably, despite limitations of secondary data availability and analysis, this study isolates discretionary screen time to a historical moment when early childhood access to screens was limited to a television, allowing us to use a simpler metric. We thus view the interpretation of our results as conservative. Given the current portability of screens and mobility from one digital tool to another, the North American guidelines clearly have reason to encourage screen-free zones, and screenfree locations at home, especially given the implications for human growth and development of young children. This research supports a strong stance for parental guidelines on availability and accessibility. Location seems to matter. 
Table 4. Unstandardized regression coefficients (standard error) reflecting the adjusted relationship between having a bedroom television at age 4 and psychological and social risks at age 12

\begin{tabular}{|c|c|c|c|c|c|}
\hline Predictor and control variables & Emotional distress & Depressive symptoms & $\begin{array}{l}B(\mathrm{SE}) \\
\text { Sociability }\end{array}$ & Victimization & Physical aggression \\
\hline Bedroom television (age 4) & $0.58(0.21)^{* *}$ & $0.67(0.25)^{* * *}$ & $-0.50(0.17)^{* *}$ & $0.39(0.20)^{*}$ & $0.38(0.14)^{* * *}$ \\
\hline Temperament (age 1.5) & $-0.11(0.14)$ & $-0.34(0.17)^{*}$ & $0.34(0.11)^{* *}$ & $-0.21(0.12)$ & $-0.07(0.09)$ \\
\hline Cognitive ability (age 2) & $0.23(0.09)^{*}$ & $-0.02(0.11)$ & $-0.21(0.08)^{* *}$ & $0.18(0.08)^{*}$ & $0.08(0.06)$ \\
\hline Screen time (age 12) & $0.02(0.01)^{* *}$ & $0.03(0.01)^{* * *}$ & $-0.02(0.01)^{* * *}$ & $0.01(0.01)$ & $0.001(0.01)$ \\
\hline Maternal education (5 mo) & $0.48(0.18)^{* *}$ & $0.75(0.20)^{* *}$ & $-0.59(0.15)^{* * *}$ & $0.27(0.15)$ & $0.26(0.13)^{*}$ \\
\hline Family dysfunction (age 1.5) & $0.14(0.14)$ & $0.10(0.16)$ & $0.01(0.12)$ & $0.08(0.13)$ & $0.02(0.09)$ \\
\hline Family configuration (age 2) & $0.12(0.22)$ & $-0.31(0.26)$ & $-0.14(0.18)$ & $0.05(0.22)$ & $0.15(0.14)$ \\
\hline Parental antisocial behavior ( $5 \mathrm{mo}$ ) & $0.12(0.13)$ & $0.03(0.16)$ & $-0.07(0.10)$ & $-0.11(0.13)$ & $-0.01(0.09)$ \\
\hline
\end{tabular}

\section{ACKNOWLEDGMENTS}

All authors have full access to all data in the study and take responsibility for its integrity and the accuracy of its analysis.

\section{FUNDING}

The Quebec Longitudinal Study of Child Development was made possible thanks to the funding provided by the Fondation Lucie et André Chagnon, the Institut de la Statistique du Québec, the Ministère de l'Éducation et de l'Enseignement supérieur (MEES), the Ministère de la Famille (MF), the Institut de recherche Robert-Sauvé en santé et en sécurité du travail (IRSST), the Centre hospitalier universitaire Sainte-Justine, and the Ministère de la Santé et des Services sociaux du Québec (MSSS). Source: Data compiled from the final master file 'E1-E20' from the Quebec Longitudinal Study of Child Development (1998-2017), @ Gouvernement du Québec, Institut de la statistique du Québec. No specific funding was received for this secondary data analysis.

\section{AUTHOR CONTRIBUTIONS}

Each author has met the Pediatric Research authorship requirements listed below. L.S. P. and M.J.H.: substantial contributions to conception and design, acquisition of data, or analysis and interpretation of data; L.S.P., M.J.H., and T.A.B.: drafting the article or revising it critically for important intellectual content; and L.S.P.: final approval of the version to be published.

\section{ADDITIONAL INFORMATION}

The online version of this article (https://doi.org/10.1038/s41390-018-0265-8) contains supplementary material, which is available to authorized users.

Competing interests: The authors declare no competing interests.

Publisher's note: Springer Nature remains neutral with regard to jurisdictional claims in published maps and institutional affiliations.

\section{REFERENCES}

1. Arem, $\mathrm{H}$. et al. Leisure time physical activity and mortality: a detailed pooled analysis of the dose-response relationship. JAMA Intern. Med. 175, 959-967 (2015).

2. American Academy of Pediatrics. Media and young minds. J. Pediatr. 138, e20162591 (2016).

3. Canadian Paediatric Society, Digital Health Task Force. Screen time and young children: promoting health and development in a digital world. J. Paediatr. Child Health 22, 461-468 (2017).

4. Pagani, L. S., Fitzpatrick, C., Barnett, T. A. \& Dubow, E. Prospective associations between early childhood television exposure and academic, psychosocial and physical well-being by middle childhood. Arch. Pediatr. Adolesc. Med. 164, 425-431 (2010).
5. Pagani, L. S., Lévesque-Seck, F. \& Fitzpatrick, C. Prospective associations between televiewing at toddlerhood and later self-reported social impairment at middle school in a Canadian longitudinal cohort born in 1997/1998. Psychol. Med. 46, 3329-3337 (2016).

6. Watt, E., Fitzpatrick, C., Derevensky, J. L. \& Pagani, L. S. Too much television? Prospective associations between early childhood televiewing and later selfreports of victimization by sixth grade classmates. J. Dev. Behav. Pediatr. 36, 426-433 (2015).

7. American Academy of Pediatrics. Media use in school-aged children and adolescents. J. Pediatr. 138, e20162592 (2016).

8. Fitzpatrick, C., Pagani, L. S. \& Barnett, T. A. Early childhood television viewing predicts explosive leg strength and waist circumference by middle childhood. Int J. Behav. Nutr. Phys. Act. 9, 87 (2012).

9. Pagani, L. S., Fitzpatrick, C. \& Barnett, T. A. Early childhood television viewing and kindergarten entry readiness. Pediatr. Res. 74, 350-355 (2013).

10. Sigman, A. Time for a view on screen time. Arch. Dis. Child. 97, 935-942 (2012).

11. Sigman, A. Screen dependency disorders: a new challenge for child neurology. J. Int Child Neurol. Assoc. 17, 119-132 (2017).

12. Takeuchi, $\mathrm{H}$. et al. The impact of television viewing on brain structures: crosssectional and longitudinal analyses. Cereb. Cortex 25, 1188-1197 (2015).

13. Meltzoff, A. N., Kuhl, P. K., Movellan, J. \& Sejnowski, T. J. Foundations for a new science of learning. Science 325, 284-288 (2009).

14. LeBlanc, A. G. et al. Correlates of total sedentary time and screen time in 9-11year-old children around the world: the international study of childhood obesity, lifestyle and the environment. PLOS ONE 10, e0129622 (2015).

15. Wansink, B. \& van Kleef, E. Dinner rituals that correlate with child and adult BMI. Obesity 22, E91-E95 (2014).

16. Cespedes, E. M. et al. Television viewing, bedroom television, and sleep duration from infancy to mid-childhood. J. Pediatr. 133, e1163-e1171 (2014).

17. Gilbert-Diamond, D., Li, Z., Adachi-Mejia, A. M., McClure, A. C. \& Sargent, J. D. Association of a television in the bedroom with increased adiposity gain in a nationally representative sample of children and adolescents. JAMA Pediatr. 168, 427-434 (2014).

18. Rideout, V. J. Measuring time spent with media: the Common Sense census of media use by US 8- to 18-year-olds. J. Child Media 10, 138-144 (2016).

19. Graham, L. T., Gosling, S. D. \& Travis, C. K. The psychology of home environments: A call for research on residential space. Perspect. Psychol. Sci. 10, 346-356 (2015).

20. Halpern D. Mental health and the built environment: more than bricks and mortar? (Abingdon-on-Thames, UK, Routledge, 2004)

21. McCormack, G. R. \& Shiell, A. In search of causality: a systematic review of the relationship between the built environment and physical activity among adults. Int J. Behav. Nutr. Phys. Act. 8, 125 (2011).

22. Gordon-Larsen P., Reynolds K. D. in Handbook of Pediatric Obesity: Etiology, Pathophysiology, and Prevention. (eds Goran M. E. \& Sothern M. S.) 251-263 (CRC Press - Taylor \& Francis Group, Boca Raton, FL, USA, 2016).

23. Pearce, M., Page, A. S., Griffin, T. P. \& Cooper, A. R. Who children spend time with after school: associations with objectively recorded indoor and outdoor physical activity. Int J. Behav. Nutr. Phys. Act. 11, 45 (2014). 
24. Christian, H. et al. Nowhere to go and nothing to do but sit? Youth screen time and the association with access to neighborhood destinations. Environ. Behav. 49, 84-108 (2017).

25. Gentile, D. A., Berch, O. N., Choo, H., Khoo, A. \& Walsh, D. A. Bedroom media: one risk factor for development. Dev. Psychol. 53, 2340-2355 (2017).

26. I am, I'll be: the survey on the future of a generation. Québec Longitudinal Study of Child Development Web site. http://www.iamillbe.stat.gouv.qc.ca/default_an. htm. Published in 2016. Accessed on 27 March 2018.

27. Dubois, L. \& Girard, M. Early determinants of overweight at 4.5 years in a population-based longitudinal study. Int J. Obes. 30, 610-617 (2006).

28. Lobstein, T. et al. Child and adolescent obesity: part of a bigger picture. Lancet 385, 2510-2520 (2015).

29. Simonato, I., Janosz, M., Archambault, I. \& Pagani, L. S. Prospective associations between toddler televiewing and subsequent lifestyle habits in adolescence. Prev. Med. 110, 24-30 (2018).

30. Kovacs, M. The Children's Depression Inventory (CDI). Psychopharmacol. Bull. 21, 995-998 (1985)

31. Robins, L. N., Helzer, J. D., Croughan, J. \& Ratcliff, K. S. The National Institute of Mental Health Diagnostic Interview Schedule: its history, characteristics, and validity. Arch. Gen. Psychiatry 38, 381-389 (1981).

32. Cummings, P. Missing data and multiple imputation. JAMA Pediatr. 167, 656-661 (2013).
33. Marsh, S., Mhurchu, C. N. \& Maddison, R. The non-advertising effects of screen-based sedentary activities on acute eating behaviours in children, adolescents, and young adults: a systematic review. Appetite 71, 259-273 (2013).

34. Higgs, S. \& Woodward, M. Television watching during lunch increases afternoon snack intake of young women. Appetite 52, 39-43 (2009).

35. Higgs, S. \& Donohoe, J. E. Focusing on food during lunch enhances food memory and decreases later snack intake. Appetite 57, 202-206 (2011).

36. Juonala, M. et al. Childhood adiposity, adult adiposity, and cardiovascular risk factors. N. Engl. J. Med. 365, 1876-1885 (2011).

37. McNaughton, S. A., Dunstan, D. W., Ball, K., Shaw, J. \& Crawford, D. Dietary quality is associated with diabetes and cardio-metabolic risk factors. J. Nutr. 139, 734-742 (2009).

38. Tremblay, M. S. et al. Canadian 24-hour movement guidelines for children and youth: an integration of physical activity, sedentary behaviour, and sleep. Appl. Physiol. Nutr. Metab. 41(6 Suppl. 3), S311-S327 (2016).

39. Hankin, B. L. et al. Depression from childhood into late adolescence: Influence of gender, development, genetic susceptibility, and peer stress. J. Abnorm. Psychol. 124, 803 (2015).

40. Patton, G. C. \& Viner, R. Pubertal transitions in health. Lancet 369, 1130-1139 (2007). 